\title{
Genistein cooperates with the histone deacetylase inhibitor vorinostat to induce cell death in prostate cancer cells
}

\author{
Cornel J Phillip', Christopher K Giardina', Birdal Bilir', David J Cutler ${ }^{5}$, Yu-Heng Lai ${ }^{1,2}$, Omer Kucuk ${ }^{3,4}$
} and Carlos S Moreno $1,2,3^{*}$

\begin{abstract}
Background: Among American men, prostate cancer is the most common, non-cutaneous malignancy that accounted for an estimated 241,000 new cases and 34,000 deaths in 2011. Previous studies have suggested that Wht pathway inhibitory genes are silenced by CpG hypermethylation, and other studies have suggested that genistein can demethylate hypermethylated DNA. Genistein is a soy isoflavone with diverse effects on cellular proliferation, survival, and gene expression that suggest it could be a potential therapeutic agent for prostate cancer. We undertook the present study to investigate the effects of genistein on the epigenome of prostate cancer cells and to discover novel combination approaches of other compounds with genistein that might be of translational utility. Here, we have investigated the effects of genistein on several prostate cancer cell lines, including the ARCaP-E/ARCaP-M model of the epithelial to mesenchymal transition (EMT), to analyze effects on their epigenetic state. In addition, we investigated the effects of combined treatment of genistein with the histone deacetylase inhibitor vorinostat on survival in prostate cancer cells.
\end{abstract}

Methods: Using whole genome expression profiling and whole genome methylation profiling, we have determined the genome-wide differences in genetic and epigenetic responses to genistein in prostate cancer cells before and after undergoing the EMT. Also, cells were treated with genistein, vorinostat, and combination treatment, where cell death and cell proliferation was determined.

Results: Contrary to earlier reports, genistein did not have an effect on CpG methylation at $20 \mu \mathrm{M}$, but it did affect histone H3K9 acetylation and induced increased expression of histone acetyltransferase 1 (HAT1). In addition, genistein also had differential effects on survival and cooperated with the histone deacteylase inhibitor vorinostat to induce cell death and inhibit proliferation.

Conclusion: Our results suggest that there are a number of pathways that are affected with genistein and vorinostat treatment such as Wnt, TNF, G2/M DNA damage checkpoint, and androgen signaling pathways. In addition, genistein cooperates with vorinostat to induce cell death in prostate cancer cell lines with a greater effect on early stage prostate cancer.

Keywords: Prostate cancer, Soy, Natural compounds, Epigenetics, Apoptosis

\footnotetext{
* Correspondence: cmoreno@emory.edu

'Department of Pathology and Laboratory Medicine, Emory University School

of Medicine, Atlanta, GA, USA

${ }^{2}$ Graduate Program in Genetics and Molecular Biology, Emory University,

Atlanta, GA, USA

Full list of author information is available at the end of the article
} 


\section{Background}

Among American men, prostate cancer is one of the most prevalent malignancies, accounting for 241,000 new cases and 34,000 deaths in 2010 [1], underscoring the importance of exploring new therapeutic approaches, targets, and the fundamental biological processes of the progression of this disease. Genistein is the major component $(50 \%)$ of soy isoflavones found in soybeans with $40 \%$ daidzein and 5-10\% glycitein [2]. Genistein has been studied extensively and shown to have exciting antitumor activities including inhibition of tyrosine kinases, angiogenesis and proliferation [3-6], telomerase activity [7], oncogene function [8], and non-specific inflammation pathways $[8,9]$, as well as induction of apoptosis $[8,10]$. The anticancer activity of genistein in vivo and in vitro has been demonstrated for carcinomas of the prostate [5,11-13], oral cavity [14], skin [15], bladder [6], and vulva [16]. Evidence suggests clearly that genistein has pleitropic effects on cancer cells, but the critical mechanisms of action remain ill defined.

Recent studies have suggested that gene promoter CpG methylation can be prevented or reversed by soy isoflavones [17]. Fang et al. reported that genistein $(2-20 \mu \mathrm{M})$ inhibited cell growth, reversed DNA hypermethylation, and reactivated RAR $\beta, \mathrm{p} 16^{\mathrm{INK} 4 \mathrm{a}}$, and MGMT in prostate cancer LNCaP and PC3 cells [17]. Genistein (20-50 $\mu \mathrm{M})$ also dose-dependently inhibited DNA methyltransferase activity, showing substrate- and methyl donor-dependent inhibition. In addition, other studies have indicated that genistein can reactivate silenced genes such as the BTG3 tumor suppressor via $\mathrm{CpG}$ demethylation and increased H3K9 histone acetylation [18-20]. These results indicate that genistein and related soy isoflavones can reactivate epigenetically silenced genes, suggesting an additional mechanism for their therapeutic effects in cancer.

Genistein is attractive as a demethylating agent and as a potential therapeutic agent compared to the nucleoside analogue 5-aza-2'-deoxycytidine (5-aza) due to its minimal toxicity. 5-aza has been shown to have some effectiveness in treating various cancer types, but side effects such as neutropenia and myelosuppression are sometimes observed in patients [21]. Genistein is a naturally occurring compound that is well tolerated with no known toxicities [22,23]. Previous studies have shown that genistein can sensitize prostate cancer cells to treatment with the chemotherapeutic drug docetaxel [8]. Despite many studies regarding genistein, its effects on demethylation and impacts on gene expression are not completely understood.

The Wnt and Notch pathways are often deregulated in prostate cancer and are important in the progression of this disease. Several negative regulators of the Wnt pathway including the adenomatous polyposis coli (APC), secreted frizzled-related protein (SFRP1), dickkopf-related protein 3
(DKK3), and sex determining region Y-box 7 (SOX7) are hypermethylated in a high proportion of prostate cancers [24-26]. In addition, another negative regulator of Wnt that reduces tumor growth, cell migration and invasion, Wnt inhibitory factor 1 (WIF1), has also been suggested to be hypermethylated in prostate cancers [27].

In this study, we tested the hypothesis that demethylation and induction of Wnt inhibitory genes by treatment with genistein might result in decreased activity of the Wnt signaling pathway. We also tested whether genistein might cooperate with other compounds such as the histone deacetylase (HDAC) inhibitor vorinostat [28] to induce apoptosis. Surprisingly, we found that contrary to earlier studies, genistein has no effect on CpG methylation at physiologically relevant concentrations using methylation specific PCR and whole genome methylation analysis. Nevertheless, we did observe that genistein affected histone acetylation and cooperates with vorinostat to induce apoptosis even better than combined treatment of vorinostat with 5-aza. Furthermore, whole genome methylation analysis and whole gene expression analysis of the effects of genistein, vorinostat, or the combination of both compounds on gene expression in ARCAP-E (epithelial) and ARCAP-M (mesenchymal) prostate cancer cells provided insights into the mechanisms of action of genistein in cells prior to and after undergoing the epithelial-to-mesenchymal transition (EMT) [29].

\section{Methods}

\section{Cell lines and prostate patient samples}

DU145, PC3, and LNCaP were obtained from the American Type Culture Collection (Manassas, VA). DU145, PC3, and LNCaP cells were maintained in Tmedia (Gibco) supplemented with 10 \% FBS, 200 mM Lglutamine, and pen-strep antibiotics. ARCaP-E and ARCaP-M cells were purchased from Novicure Biotechnology and propagated in MCaP-Medium (Novicure Biotechnology, Inc, Birmingham, AL), supplemented with $5 \%$ FBS, $200 \mathrm{mM}$ L-glutamine, and pen-strep. Cells were counted on a hemocytometer after staining with Trypan Blue. Additionally, prostate patient samples from primary tumors were obtained under IRB approved protocols from Emory University Hospital. Clinical characteristics of these samples are provided in Supplemental Table S1 (Additional file 1: Table S1.)

\section{Materials}

Genistein and 5-aza-2'-deoxycytidine (5-aza) were obtained from Sigma Aldrich (St. Louis, MO), ICG-001 was obtained as a gift from the University of Southern California in the laboratory of Dr. Michael Kahn, University of Southern California, N-[N-(3,5-Difluorophenacetyl)-L-alanyl]-(S)-phenylglcine t-butyl ester (DAPT) was obtained from Santa Cruz Biotechnology, Inc (Santa 
Cruz, California), and N-Hydroxy-N'-phenyloctanediamide (vorinostat) was obtained from Toronto Research Chemicals, Inc (North York, ON, Canada). Each drug was dissolved in dimethylsulfoxide (DMSO) and stored in aliquots at $-20^{\circ} \mathrm{C}$.

\section{Methylation specific PCR (MSP)}

DNA was extracted using the DNeasy Blood and Tissue Kit (Qiagen, Valencia, CA). Bisulfite treatment was performed using the EZ DNA Methylation Direct kit (Zymo Research Corp, Orange, CA) and MSP was performed using the EZ DNA Methylation startup kit (Zymo Research Corp, Orange, CA). Methylated and unmethylated primers were designed and optimized for APC, DKK3 SOX7, WIF1, SFRP1, and SFRP2 (Additional File 1: Table S2). In addition, the size of the product and the annealing temperature for each primer pair are indicated.

\section{Chromatin immunoprecipitation (ChIP) assay}

ChIP assays were performed as described previously [30] using $90 \%$ confluent ARCaP-E cells, fixed in $1 \%$ formaldehyde and sonicated for 10 minutes. Sonicated chromatin was immunoprecipitated with $\mathrm{H} 3$ acetyl K9 antibody (Abcam, San Francisco, CA,USA) and rabbit IgG (Vector Laboratories, Burlingame, CA, USA) and collected with protein $\mathrm{G}$ agarose beads (Upstate, Temecula, CA, USA). Cells were washed twice with IP Dilution Buffer, TSE-500, LiCl Detergent, and TE buffer in listed order. Beads were then eluted and PCR was performed on the purified DNA using the primers in Additional File 1: Table S3.

\section{Cell death assay}

After indicated drug treatments, cells were trypsinized and washed twice with $1 \mathrm{X}$ PBS. Cells were subsequently resuspended in 1X Annexin $\mathrm{V}$ binding buffer and incubated with Annexin V-FITC (Pharmingen Biosciences, San Diego, CA, USA) for 15 minutes at room temperature. $400 \mu \mathrm{l}$ of Annexin binding buffer and $1 \mu \mathrm{g} / \mathrm{mL}$ propidium iodide (PI) were then added. The total cell death was measured using the BD FACSCalibur system (BD Biosciences Pharmingen, San Diego, CA, USA).

\section{Quantitative real time RT-PCR}

Total RNA was isolated from cells using the RNeasy Mini Kit (Qiagen, Valencia, CA). After RNA isolation, total RNA was reverse transcribed to CDNA and quantitative real-time PCR was performed using the iQ SYBER Green Supermix on a Bio-Rad iCycler in a $25 \mu \mathrm{l}$ total volume reaction. Primer sequences for Real-time RTPCR are listed in Additional file 1: Table S4. The relative gene expression levels were determined by the $\Delta \Delta C_{T}$ method comparing threshold cycles with $\beta$-actin or $18 \mathrm{~S}$ as a normalization control.

\section{Bisulfite sequencing}

Samples were sequenced on an Ion Torrent Personal Genome Machine at the Cancer Genomics Shared Resource at the Winship Cancer Institute. Sample DNAs (600 ng), which had been previously used for the whole genome methylation analysis, were bisulfite modified using the EZ DNA Methylation-Direct kit (Zymo Research) as before. Methylation-specific primers were designed using MethPrimer software [31] to amplify a 175-bp region of the WIF1 promoter (Forward: AATAGTTTTGGTTGAGGGAGTTGTA, Reverse: ACCAACAAACACAAAAAAATACTCC). PCR reaction was performed using $1 \mu \mathrm{l}$ of bisulfite-treated DNA templates, 1 X Zymo Taq PreMix (Zymo Research), and $0.4 \mu \mathrm{M}$ of each primer in a total volume of $25 \mu \mathrm{l}$. Cycling parameters for PCR were $95^{\circ} \mathrm{C}$ $5 \mathrm{~min}$, followed by 40 cycles of $95^{\circ} \mathrm{C} 30 \mathrm{~s}, 58.2^{\circ} \mathrm{C} 30 \mathrm{~s}$, and $72^{\circ} \mathrm{C} 30 \mathrm{~s}$, followed by the extension step at $72^{\circ} \mathrm{C} 10 \mathrm{~min}$, and $4^{\circ} \mathrm{C}$ hold. Amplicons were gel-extracted using the Qiagen Gel Purification kit (Qiagen), and submitted to the Emory Winship Cancer Institute Cancer Genomics Shared Resource for Ion Torrent sequencing, which was performed as described [32]. Sequencing data was analyzed using PEMapper software (Cutler D, Patel V, Mondal K, Ramachandran D, Steinberg K, Shetty A, Zwick M: PEMapper: A New Approach to Identifying Genetic Variation in Second-Generation Sequencing Studies, submitted).

\section{Whole genome methylation profiling}

DNA was isolated from three independent experiments in which ARCaP-E and ARCaP-M cells were treated with DMSO control, $20 \mu \mathrm{M}$ genistein, or $1 \mu \mathrm{M}$ 5-aza for six days. Fresh drugs and media were changed every other day. Additionally, PREC cells (Lonza, Basel, Switzerland) were used as a normal human prostate cell line control. Total DNA was submitted to the Emory Winship Cancer Institute Cancer Genomics Shared Resource for analysis with Illumina $27 \mathrm{~K} \mathrm{CpG}$ Methylation Arrays that interrogate 27,578 CpG loci > 14,000 genes using the Illumina Beadstation 500 instrument. After data normalization, Genome Studio software (Illumina) was used to compute $\beta$ values (range $0-1$ ) defined as $\beta=$ methylated signal/(methylated + unmethylated signal). Probes were filtered to include only those with changes in $\beta$ values $>0.2$, and significance analysis of microarrays (SAM) software [33] was used to determine statistically significant changes in methylation. SAM analysis was completed with two-class unpaired settings, 500 permutations, and FDR $<1 \%$.

\section{Whole genome expression profiling}

RNA was isolated from three independent experiments in which ARCaP-E and ARCaP-M were treated with DMSO control, $20 \mu \mathrm{M}$ genistein for 6 days, vorinostat for $48 \mathrm{hrs}$, or a combination of vorinostat (48 hrs) plus genistein 
(6 days). Total RNA was submitted to the Emory Winship Cancer Institute Cancer Genomics Shared Resource for processing and hybridization to Illumina Human HT-12 v3 Expression BeadChips that interrogate 48,804 probes. After hybridization and data normalization, significance analysis of microarrays (SAM) software was used to determine significant differences in gene expression. SAM settings were two class unpaired, 500 permutations, and a minimum fold change of 1.5 at FDR $<1 \%$.

\section{Immunoblotting}

DMSO and genistein treated cells were collected and centrifuged at $1000 \mathrm{rpm}$ for 5 minutes, and the pellets were washed twice with $1 \mathrm{X}$ PBS. Cells were lysed using lysis buffer. Lysis buffer consisted of $0.137 \mathrm{~mol} / \mathrm{L} \mathrm{NaCl}, 0.02 \mathrm{~mol} / \mathrm{L}$ TRIS (pH 8.0), $10 \%$ glycerol, 1 \% NP40, and a protease inhibitor cocktail obtained from Promega (San Luis Obispo, CA, USA). A mix of cell lysates $(50 \mu \mathrm{g})$ and laemmli sample buffer (Bio Rad, Hercules, CA) were made and then heated at $99^{\circ} \mathrm{C}$ for 3 minutes. Proteins were separated by SDSPAGE electrophoresis and transferred to nitrocellulose for immunoblotting. After transfer, nitrocellulose membrane was blocked for $1 \mathrm{hr}$ at room temperature using blocking buffer from Li-Cor Biosciences (Lincoln, NE, USA). Membrane was incubated with primary antibody Histone acetyltransferases 1 (HAT1) (Santa Cruz, Santa Cruz, CA) overnight at $4^{\circ} \mathrm{C}$ and then washed three times with $1 \mathrm{X}$ PBS and $0.1 \%$ Tween. To control for equal loading, GAPDH (Cell Signaling, Danvers, MA) was used as a loading control. Then the nitrocellulose membrane was incubated with a secondary fluorescent antibody (LI-COR Biosciences, Lincoln, NE, USA). Using the manufacturer's protocol, membranes were imaged and quantitated using the Odyssey imaging system (LI-COR Biosciences, Lincoln, NE, USA).

\section{Results}

\section{Wnt inhibitory genes are methylated in prostate cancer} patient samples

Tumor suppressor genes are often hypermethylated in prostate cancer patient tissue samples compared to normal tissues, and this methylation can correlate with prognosis $[34,35]$. To determine if Wnt Inhibitory genes are methylated in prostate cancer patient samples, we performed methylation specific PCR (MSP) on eight prostate cancer patient samples. We observed that SOX7 was highly methylated, whereas WIF1, SFRP1, DKK3, and APC were partially methylated in each of these samples (Figure 1A).

\section{Genistein treatment does not induce demethylation of wnt-inhibitory genes but does induce expression and $\mathrm{H} 3 \mathrm{~K} 9$ acetylation in prostate cancer cells}

To test our hypothesis that genistein could demethylate Wnt inhibitory genes, APC, SOX7, SFRP1, DKK3, and
WIF1 were tested for demethylation by MSP in DU145, PC-3, and ARCaP-E cells following treatment for 6 days with $20 \mu \mathrm{M}$ genistein or with 5 -aza as a positive control. Although some studies have used concentrations as high as $50 \mu \mathrm{M}$ [20], other previously published studies have used $20 \mu \mathrm{M}$ [17], and analysis of genistein concentrations in the prostates of patients supplemented with $82 \mathrm{mg} /$ day determined that the median concentration of genistein in the prostate was only $2.3 \mu \mathrm{M}$, suggesting that achieving $50 \mu \mathrm{M}$ genistein in patients is likely not attainable [23]. 5aza treated cells demonstrated significant demethylation of SOX7 in PC3 and DU145 cells (Additional File 2: Figure S1), confirming the sensitivity of the MSP assay. Although previous reports indicated that genistein has the potential to demethylate $\mathrm{CpG}$ dinucleotides [17,20], we observed no demethylation of APC, SOX7, WIF1, or SFRP1 in DU145, PC3, or ARCaP-E cells when treated with $20 \mu \mathrm{M}$ genistein (Figure $1 \mathrm{~B}$ and Additional File 2: Figure S1). In addition, we performed whole genome methylation profiling of ARCaP-E and ARCaP-M cells treated with DMSO, $1 \mu \mathrm{M} 5$-aza, or $20 \mu \mathrm{M}$ genistein for 6 days. Consistent with the MSP data, we observed no significant changes in methylation following genistein treatment across over 14,000 genes tested by this platform (Figure $1 \mathrm{C}$ and Additional File 2: Figure S1). In contrast, ARCaP-E and ARCaP-M cells treated with $1 \mu \mathrm{M}$ 5-aza, exhibited a substantial change in methylation. Furthermore, bisulfite sequencing was performed on $13 \mathrm{CpGs}$ over 175 basepairs of the WIF1 CpG island to obtain over 1000 reads per genomic DNA sample by next generation sequencing methods on an Ion Torrent Personal Genome Machine. Analysis of these 13 CpGs in the WIF1 CpG island in ARCAP-E, ARCAP-M, and PrEC cells indicated no change in CpG methylation upon genistein treatment (Figure 1D). Thus, we conclude that treatment with $20 \mu \mathrm{M}$ genistein for 6 days does not induce CpG demethylation in prostate cancer cells.

It has also been previously reported that genistein can affect histone acetylation [18,36]. Consequently, we tested the effect of genistein by ChIP assay and observed that it did produce substantial changes in H3K9 acetylation in the promoters of Wnt inhibitory genes. ARCaPE cells treated with genistein for 6 days at $20 \mu \mathrm{M}$ demonstrated an increase in acetylation in SOX7, APC, DKK3, WIF1, SFRP1, and SFRP2 (Figure 1E). Additionally, there was an increase in the histone acetyltransferase 1 (HAT1) protein when treated with genistein (Figure 1F).

To determine if genistein treatment would induce gene expression of Wnt inhibitory genes, we performed quantitative real time PCR (QPCR) to determine if there was an increase in the mRNA levels of SOX7, SFRP2, SFRP1, APC, and DKK3. We did not observe any significant increases in ARCAP-E cells following genistein treatment, although there was a small but significant 


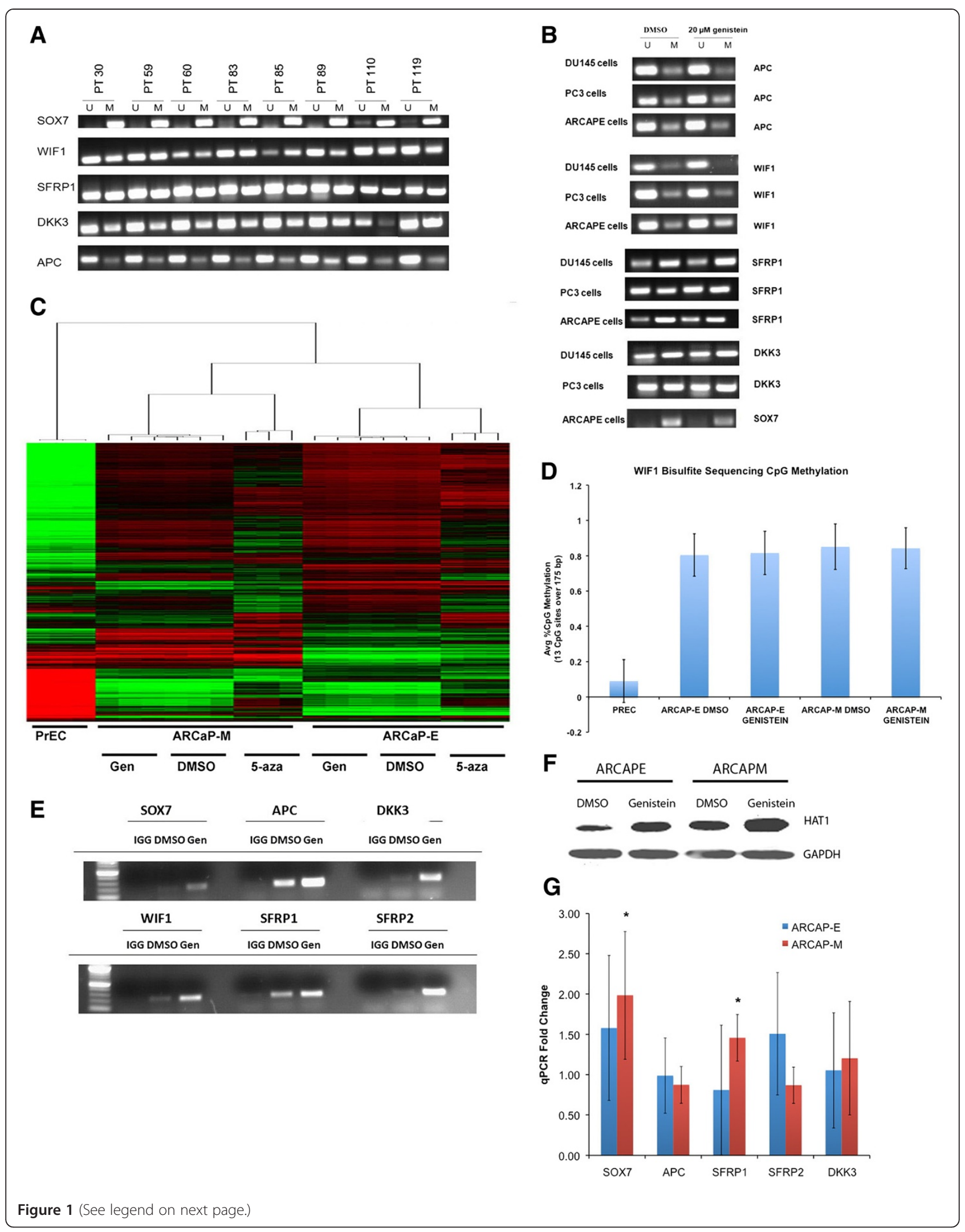


(See figure on previous page.)

Figure 1 Genistein treatment induces hypermethylated Wnt-inhibitory genes via H3K9 acetylation, not CpG demethylation. (A) Wnt Inhibitory genes are methylated in prostate cancer patient samples. MSP of Wnt inhibitory genes was performed on genomic DNA derived from paraffin embedded prostate tissues in 8 prostate cancer samples. SOX7 was strongly methylated while WIF1, SFRP1, DKK3, and APC were partially methylated in multiple patient samples. $U=$ unmethylated, $M=$ methylated. (B) MSP analysis indicates no change in methylation status of APC, WIF1, SFRP1, SOX7, and DKK3 in DU145, ARCAPE, and PC3 cell lines after treatment with $20 \mu$ M genistein for 6 days. (C) Whole genome methylation profiling using the $27 \mathrm{~K} \mathrm{CpG}$ Methylation Arrays was performed on ARCAPE and ARCAPM cells treated with DMSO and $20 \mu \mathrm{M}$ genistein for 6 days. In addition, 5-aza-deoxy-cytidine was used as a positive control and PREC cells were used as a negative control. No significant changes in methylation were detected with genistein treatment. Unsupervised hierarchical clustering of 4,190 CpG loci with $\beta$-value $>$ 0.5 is shown. (D) Bisulfite sequencing of 175 bp of the WIF1 CpG island across 13 CpG sites indicates high methylation in ARCAP-E and ARCAP-M cells with or without genistein treatment, and low methylation in PrEC cells. (E) Anti-acetyl histone H3-Lysine9 chromatin immunoprecipitation (acetyl-H3K9 ChIP) of ARCAP-E cells treated with DMSO control or genistein shows marked increases in acetyl-H3K9 following genistein treatment. (F) Immunoblot of HAT1 protein shows increased HAT1 protein in ARCaP-E and ARCaP-M after genistein treatment. (G) Gene expression with genistein treatment in ARCAPE, and ARCAPM cells. Gene expression of indicated Wnt inhibitory genes after treatment with genistein was determined using QPCR. The data are presented as fold change relative to DMSO control (mean \pm SD, triplicate samples from five independent experiments). Significant $p$-values $(p<0.05)$ were computed using the student's $t$-test with a two-tailed distribution and are indicated with an asterisk $\left(^{*}\right)$.

increase in SOX7 and SFRP1 expression in ARCAP-M cells (Figure 1G).

\section{Genistein treatment reduces proliferation and induces apoptosis in prostate cancer cells alone or in combination with vorinostat}

To determine genistein's potential as a therapeutic agent in the treatment of prostate cancer, prostate cancer cell lines PC3, DU145, ARCaP-E, ARCaP-M and LNCaP were treated with $20 \mu \mathrm{M}$ genistein for a total of six days, $1 \mu \mathrm{M}$ vorinostat for 2 days, and a combination of genistein and vorinostat (Figure 2A). Since genistein treatment increased histone acetylation, we hypothesized that it might cooperate with histone deacetylase (HDAC) inhibitors to induce apoptosis. Genistein exhibited only a minor (5-10\%) effect of increased cell death on these cells based on Annexin V/PI staining (Figure 2A). There was an approximate increase of $8 \%$ cell death in DU145 cells, $5 \%$ cell death in ARCaP-E cells, $10 \%$ cell death in LNCaP cells, and $8 \%$ cell death in PC3 cells when compared to untreated DMSO cohorts. Nevertheless, we confirmed previous studies $[37,38]$ indicating that genistein was quite effective in inhibiting cell proliferation (Figure 2B). In addition, there was an increase in cell death of all prostate models when treated with vorinostat and combination genistein and vorinostat with the largest affect being in the ARCaP-E cell line model.

To compare the effectiveness of genistein and 5-aza, ARCaP-E and ARCaP-M cells were treated with either $20 \mu \mathrm{M}$ genistein or $1 \mu \mathrm{M} 5$-aza, in combination with the HDAC inhibitor vorinostat for $48 \mathrm{hrs}$. Combination treatment of vorinostat and genistein showed that there was a more than additive effect on cell death in both ARCaP cell lines (Figure 2C). Also, these data suggest that prostate cancer cells that undergo EMT may become less sensitive to vorinostat treatment. Although 5-aza, as a single agent, increased cell death more than genistein as a single, when 5aza was used in combination with vorinostat, there was no substantial increase in cell death. Interestingly, in ARCaP-E cells, the combined 5-aza and vorinostat actually produced less cell death than both agents used separately. In addition, genistein combined with vorinostat was much more effective than 5-aza combined with vorinostat in inducing cell death (Figure 2C). This is potentially of significant clinical relevance because genistein has no known toxicities.

In addition to genistein's effects on cell death, we also measured genistein's effects on cellular proliferation. In ARCaP-E cells, we observed that genistein was more effective in inhibiting cell growth than either vorinostat or 5-aza (Figure 2D). There was greater than $60 \%$ and $50 \%$ inhibition of growth in ARCaP-E and ARCaP-M cell lines, respectively, following genistein treatment. ARCaP-E cells treated with 5 -aza showed only a $35 \%$ reduction in proliferation, whereas ARCaP-M cells had a greater than $50 \%$ inhibition of growth with 5-aza alone (Figure 2D). Importantly, the combination of genistein and vorinostat decreased proliferation by $80 \%$ in ARCaP-E and greater than $60 \%$ in ARCaP-M cells. These data show that genistein is more effective than 5-aza in inhibiting cell proliferation in ARCAP-E cells, and that combined genistein and vorinostat has a profound inhibition on cellular proliferation.

\section{DNA damage checkpoint and apoptosis networks identified by whole genome expression profiling of cells treated with genistein and vorinostat}

To determine the genome-wide effects of genistein, vorinostat, and combined treatment on gene expression, we conducted whole genome expression profiling of ARCaP$\mathrm{E}$ and ARCaP-M cells using Illumina HT-12 v3 Expression BeadChips. Genistein treatment had a larger effect on ARCaP-E cells (291 genes induced and 144 genes repressed) than on ARCaP-M cells (31 genes induced and 33 repressed). Vorinostat impacted more genes than genistein in both ARCaP-E cells (820 genes induced and 1046 genes repressed) and ARCaP-M cells (1296 genes induced and 883 genes repressed). As expected, the largest changes 


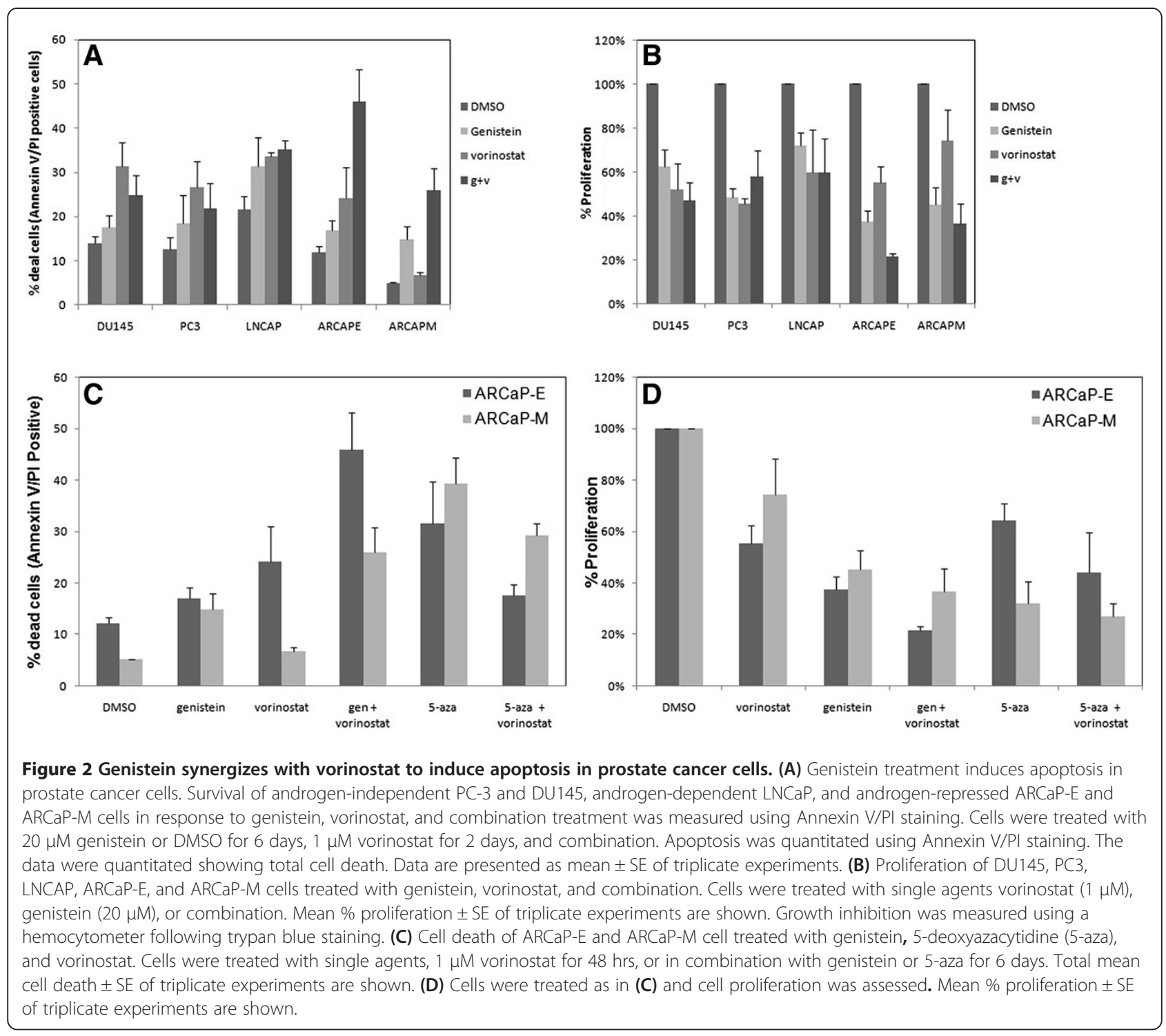

in gene expression were observed by combined treatment with genistein and vorinostat for ARCaP-E cells (1978 genes induced and 1758 genes repressed) and ARCaP-M cells (1503 genes induced and 1161 genes repressed) (Figure 3A). Gene ontology enrichment analysis using the DAVID knowledgebase [39] and Ingenuity Pathway Analysis (IPA) [40], demonstrated that the affected genes were highly enriched in genes involved in DNA damage, cell cycle arrest, and apoptosis (Tables 1 and 2). Interestingly, IPA analysis of genes affected by combined genistein and vorinostat treatment identified a gene network with the pro-apoptotic Tumor Necrosis Factor alpha (TNF $\alpha$ ) as a major hub (Figure 3B), including the pro-survival gene BIRC7 (or Livin) which was downregulated 4.8-fold. In addition, genes involved in the $\mathrm{G} 2 / \mathrm{M}$ cell cycle and response to DNA damage was also identified (Figure 3C-D). For example, four members of the minichromosome maintenance complex (MCM) essential for DNA replication are strongly upregulated, as are BRCA1, BARD1, RAD23B, and XRCC2. QPCR analysis following genistein treatment confirmed reduced levels of BIRC7, as well as SLUG, HES1, and TGFB1I1 (or ARA55), and several genes associated with apoptosis (Figure 3E). These data indicate that genistein affects cell survival and proliferation via multiple mechanisms including the TNF $\alpha-\mathrm{NF}_{\mathrm{K} B}$ and ATM-CHEK2-BRCA1 pathways. Additionally, genes involved in chromatin modifications and histone acetylations such as HAT1 were also impacted by genistein treatment (Table 1 and Additional File 3: Figure S2).

\section{Discussion}

Genistein is a pleiotropic compound with many clinically attractive properties. Previous studies have suggested that some of the mechanisms of action of genistein includes 


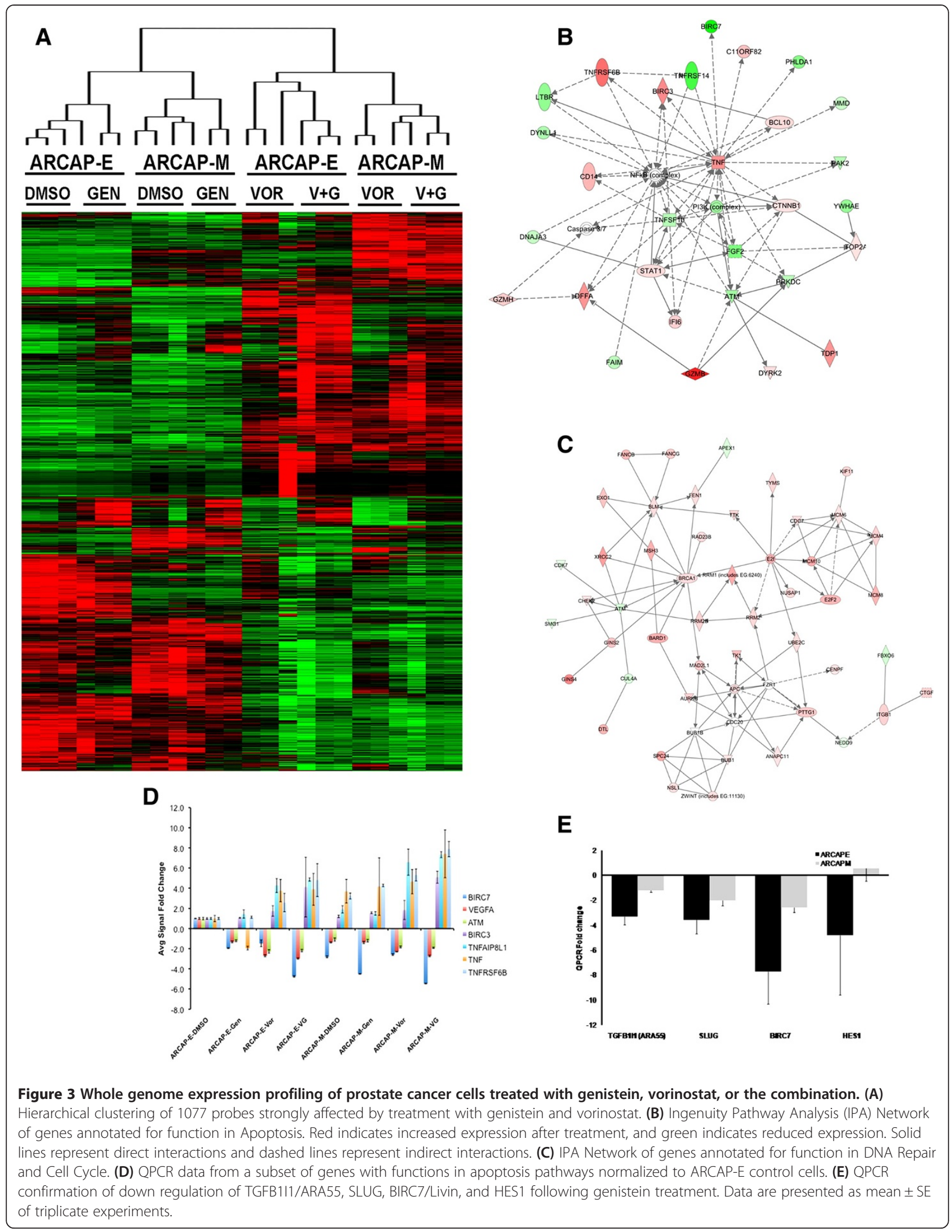


Table 1 Fold changes in ARCAP-E cells (combined vorinostat/genistein vs. DMSO) of critical genes involved in apoptosis, DNA damage checkpoint, and chromatin structure and remodeling

\begin{tabular}{llllllll}
\hline Symbol & Synonym & Fold Change & Network & Symbol & Synonym & Fold Change & Network \\
\hline GZMB & Granzyme B & 6.9 & Apoptosis & CTNNB1 & $\beta$-catenin & 1.7 & Apoptosis \\
\hline TNFRSF6B & Decoy Receptor 3 & 4.8 & Apoptosis & MTA2 & - & 1.7 & Chromatin \\
\hline BIRC3 & C-IAP2 & 4.1 & Apoptosis & CHEK2 & RAD53 & 1.6 & DNA Checkpoint \\
\hline TNF & TNFa & 3.9 & Apoptosis & BUB1 & - & 1.6 & DNA Checkpoint \\
\hline DFFA & ICAD & 3.8 & Apoptosis & TGFB111 & ARA55 & -1.8 & Apoptosis \\
\hline BARD1 & BRCA1 Associated & 3.0 & DNA Checkpoint & TNFSF10 & TRAlL & -1.9 & Apoptosis \\
\hline MAD2L1 & - & 2.4 & DNA Checkpoint & CREBBP & CBP & -2.1 & Chromatin \\
\hline HAT1 & Histone Acetyltrans-ferase 1 & 2.4 & Chromatin & SIRT1 & SIR2L1 & -2.1 & Chromatin \\
\hline GZMH & Granyme H & 2.3 & Apoptosis & ATM & TEL1 & -2.2 & DNA Checkpoint \\
\hline AURKB & Aurora Kinase B & 2.2 & DNA Checkpoint & TNFRSF14 & LIGHTR & -3.7 & Apoptosis \\
\hline BCL10 & - & 2.0 & Apoptosis & BIRC7 & Livin & -4.8 & Apoptosis \\
\hline BRCA1 & - & 1.9 & DNA Checkpoint & & & &
\end{tabular}

inhibition of tyrosine kinases and NFkB, DNA CpG demethylation, and other mechanisms [3,5,6,18]. Initially, we hypothesized that genistein would demethylate Wnt inhibitory genes and induce their expression, possibly inhibiting the Wnt pathway. Although previous studies showed that genistein demethylates $\mathrm{CpG}$ dinucleotides at $50 \mu \mathrm{M}$ [20], our experiments showed no effect at $20 \mu \mathrm{M}$. Moreover, analysis of genistein concentrations in the prostates of patients supplemented with $82 \mathrm{mg} /$ day determined that the median concentration of genistein in the prostate was only $2.3 \mu \mathrm{M}$ [23]. Despite the fact that genistein has a low toxicity and is well tolerated at high concentrations in individuals, high concentrations of genistein that are capable of demethylating DNA in a physiological setting may not be clinically feasible. Nevertheless, genistein may still prove to be useful clinically as a chemopreventative or as a therapeutic agent in combination therapy with drugs such as vorinostat, since combining genistein with vorinostat demonstrated a more than additive effect on inducing cell death (Figure 2A). Genistein was also tested in combination with a Notch inhibitor (DAPT), Wnt inhibitor (ICG-001), and an AKT inhibitor (LY294002). However, those treatments in combination did not prove to be as effective in inducing cell death in prostate cancer cells as combination genistein and vorinostat (Additional File 4: Figure S3).
Previous studies have reported that genistein reduces cell growth and induces apoptosis in a number of cancer cells [8]. Our data suggest that this may be as a result of increase in genes that affect the G2/M checkpoint such as BRCA1, BARD1, BUB1, AURKB, CHEK2, and MAD2L1 as well as genes involved in apoptosis such as GZMB, DFFA, TNF, BIRC3, BCL10, and BIRC7/Livin (Tables 1 and 2). These data provide evidence for potential mechanisms to explain earlier studies showing that genistein sensitizes prostate cells to treatment with docetaxel and selenium $[41,42]$.

Our data from whole genome expression analysis and real time PCR validation suggest that the mechanism of action of cell death from genistein may be due to down regulation of anti-apoptotic genes such as BIRC7/Livin, TGFB1I1/ARA55, HES1, and SLUG that are involved in the TNF-NFKB and the androgen pathway (Figure 3). SLUG (a.k.a. SNAI2) plays a critical role in mediating the EMT [43] along with SNAIL [44], the founding member of this family of transcription factors. HES1 is a downstream target and mediator of the Notch signaling pathway [45], suggesting that genistein treatment may inhibit Notch pathway activity. TGFB1I1/ARA55 is induced by TGB $\beta$ signals and acts as a co-activator for the androgen receptor [46], suggesting that both TGB $\beta$ signaling and androgen signaling may be inhibited by

Table 2 Gene ontology analysis of genes affected by combined genistein and vorinostat treatment using DAVID knowledgebase (columns 1-4) and ingenuity knowledgebase (columns 5-7)

\begin{tabular}{lllllll}
\hline GO Term & Biological Process & Count & $\boldsymbol{p}$-value & IPA Biological Function & Count & $\boldsymbol{p}$-value \\
\hline GO:0006281 & DNA repair & 45 & $1.03 \mathrm{E}-13$ & DNA Replication, Recombination, and Repair & 39 & $7.18 \mathrm{E}-12$ \\
\hline GO:0008219 & Cell Death & 48 & $2.73 \mathrm{E}-03$ & Cell Death & 122 & $1.95 \mathrm{E}-10$ \\
\hline GO:0022403 & Cell Cycle & 57 & $1.63 \mathrm{E}-14$ & Cell Cycle & 105 & $2.70 \mathrm{E}-09$ \\
\hline GO:0006915 & Apoptosis & 43 & $1.37 \mathrm{E}-03$ & Apoptosis & 149 & $1.13 \mathrm{E}-07$ \\
\hline GO:0000075 & Cell Cycle Checkpoint & 17 & $1.45 \mathrm{E}-06$ & DNA checkpoint control & 13 & $1.90 \mathrm{E}-06$ \\
\hline GO:0006325 & Chromatin Organization & 28 & $7.01 \mathrm{E}-03$ & Binding Of Chromatin & 5 & $1.69 \mathrm{E}-03$ \\
\hline
\end{tabular}


genistein. We observed that ARCaP-E cells are more sensitive to genistein and vorinostat treatment than ARCaP-M cells (Figure 2C), and we also determined that there was a greater decrease in BIRC7/Livin, TGFB1I1/ ARA55, HES1, and SLUG in ARCaP-E cells compared to ARCaP-M cells (Figure 3E), suggesting possible mechanisms for chemotherapeutic resistance in mesenchymal cells compared to epithelial cells.

\section{Conclusion}

The effects of genistein treatment on epigenetics and gene expression are likely due primarily to changes in histone acetylation rather than $\mathrm{CpG}$ methylation. Similar to previous reports $[19,20]$ we observed an increase of HAT1 upon genistein treatment in our array data (Table 1) and increase protein levels of HAT1 (Figure 1E). This change in HAT1 expression may provide a mechanism for increased H3K9 acetylation and explain why Wnt inhibitory genes such as SOX7 were slightly induced when treated with genistein despite a lack of change in CpG methylation. We observed changes in a large number of genes and pathways that were affected with combinatorial effects of genistein and vorinostat including the TNFNFkB pathway and G2/M cell cycle arrest in response to DNA damage and repair (Figure $3 \mathrm{C}$ and Tables 1 and 2).

In conclusion, we have shown that genistein can cooperate with vorinostat to induce apoptosis, however, future studies are needed to validate this combination in a clinical setting.

\section{Additional files}

Additional file 1: Table S1. Clinical characteristics of patient samples.

Table S2. Primer sequences for methylated and unmethylated genes.

Table S3. Primer sequences for ChIP assay. Table S4. Primer sequences for Real time RT PCR

Additional file 2: Figure S1. (A) DU145 and PC3 cells were treated with increased doses 5-deoxy-azacytidine or genistein, and methylation of SOX7 was assessed by MSP. Although there is an apparent slight decrease in methylated SOX7 in PC3 cells, there is no corresponding increase in unmethylated SOX7 in these cells. Moreover, there is no change in SOX7 methylation in DU145 cells. (B) Histogram of beta-values representing the global level of $\mathrm{CpG}$ methylation in the ARCaP-E cell model when treated with DMSO, genistein, or 5-aza.

Additional file 3: Figure S2. Network of genes annotated for function in chromatin structure and remodeling.

Additional file 4: Figure S3. (A) Combination cell death with genistein for 6 days in combination with $5 \mu \mathrm{M}$ vorinostat, $10 \mu \mathrm{M}$ DAPT, $10 \mu \mathrm{M}$ ICG-

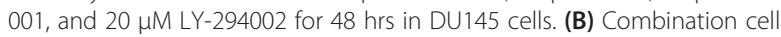
death with genistein for 6 days in combination with $5 \mu \mathrm{M}$ vorinostat, $10 \mu \mathrm{M}$ DAPT, $10 \mu \mathrm{M}$ ICG-001, and $20 \mu \mathrm{M}$ LY-294002 for 48 hrs in ARCaPE cells.

\section{Competing interests}

The authors declare that they have no competing interests.

\section{Authors' contributions}

The authors contributions are the following: CJP contributed with experimental design, collection and analysis of data, interpretation of findings, and writing of the manuscript. CKG contributed with experimental design, collection and analysis of data, and editing of the manuscript. YL contributed with collection and analysis of the data and editing of the manuscript. DJC performed Ion Torrent Data sequence analysis. OK contributed with experimental design, provided prostate patient samples, and editing of the manuscript. CSM contributed with experimental design, analysis of data, interpretation of findings, and editing of the manuscript. All authors read and approved the final manuscript.

\section{Acknowledgements}

Grant support: National Cancer Institute 2R01 CA 106826 (C.S. Moreno), Research Supplements to Promote Diversity in Health-Related Research grant R01CA106826-06 S1 (C.J. Phillip). In addition, this research was supported in part by funds from Georgia Cancer Coalition. Omer Kucuk is a Distinguished Scholar of Georgia Cancer Coalition, and Chris Giardina is Georgia Cancer Coalition Carpenter Fellow.

We thank Emory Winship Cancer Institute Cancer Genomics Shared Resource supported by NCl Cancer Center Support Grant P30CA138292 for performing Illumina gene expression arrays, Illumina CpG methylation arrays, and next generation bisulfite sequencing.

\section{Author details}

'Department of Pathology and Laboratory Medicine, Emory University School of Medicine, Atlanta, GA, USA. ${ }^{2}$ Graduate Program in Genetics and Molecular Biology, Emory University, Atlanta, GA, USA. ${ }^{3}$ Winship Cancer Institute, Emory University, Atlanta, GA, USA. ${ }^{4}$ Department of Hematology and Medical Oncology, Emory University School of Medicine, Atlanta, GA, USA.

${ }^{5}$ Department of Human Genetics, Emory University, Atlanta, GA, USA.

Received: 10 August 2011 Accepted: 23 March 2012

Published: 11 April 2012

\section{References}

1. Trapnell C, Williams BA, Pertea G, Mortazavi A, Kwan G, van Baren MJ, Salzberg SL, Wold BJ, Pachter L: Transcript assembly and quantification by RNA-Seq reveals unannotated transcripts and isoform switching during cell differentiation. Nat Biotechnol 2010, 28(5):511-515.

2. Delmonte P, Perry J, Rader Jl: Determination of isoflavones in dietary supplements containing soy, Red Clover and kudzu: extraction followed by basic or acid hydrolysis. J Chromatogr A 2006, 1107(1-2):59-69.

3. Sasamura H, Takahashi A, Yuan J, Kitamura H, Masumori N, Miyao N, Itoh N, Tsukamoto T: Antiproliferative and antiangiogenic activities of genistein in human renal cell carcinoma. Urology 2004, 64(2):389-393.

4. Zhou JR, Gugger ET, Tanaka T, Guo Y, Blackburn GL, Clinton SK: Soybean phytochemicals inhibit the growth of transplantable human prostate carcinoma and tumor angiogenesis in mice. J Nutr 1999, 129(9):1628-1635.

5. Hillman GG, Wang Y, Kucuk O, Che M, Doerge DR, Yudelev M, Joiner MC Marples B, Forman JD, Sarkar FH: Genistein potentiates inhibition of tumor growth by radiation in a prostate cancer orthotopic model. Mol Cancer Ther 2004, 3(10):1271-1279.

6. Singh AV, Franke AA, Blackburn GL, Zhou JR: Soy phytochemicals prevent orthotopic growth and metastasis of bladder cancer in mice by alterations of cancer cell proliferation and apoptosis and tumor angiogenesis. Cancer Res 2006, 66(3):1851-1858.

7. Jagadeesh S, Kyo S, Banerjee PP: Genistein represses telomerase activity via both transcriptional and posttranslational mechanisms in human prostate cancer cells. Cancer Res 2006, 66(4):2107-2115.

8. Li Y, Ahmed F, Ali S, Philip PA, Kucuk O, Sarkar FH: Inactivation of nuclear factor kappaB by soy isoflavone genistein contributes to increased apoptosis induced by chemotherapeutic agents in human cancer cells. Cancer Res 2005, 65(15):6934-6942.

9. Ye F, Wu J, Dunn T, Yi J, Tong X, Zhang D: Inhibition of cyclooxygenase-2 activity in head and neck cancer cells by genistein. Cancer Lett 2004, 211 (1):39-46.

10. Li Y, Ellis KL, Ali S, El-Rayes BF, Nedeljkovic-Kurepa A, Kucuk O, Philip PA, Sarkar FH: Apoptosis-inducing effect of chemotherapeutic agents is potentiated by soy isoflavone genistein, a natural inhibitor of NF-kappaB in BxPC-3 pancreatic cancer cell line. Pancreas 2004, 28(4):e90-e95.

11. Wang Y, Raffoul JJ, Che M, Doerge DR, Joiner MC, Kucuk O, Sarkar FH, Hillman GG: Prostate cancer treatment is enhanced by genistein in vitro 
and in vivo in a syngeneic orthotopic tumor model. Radiat Res 2006, 166 (1 Pt 1):73-80

12. Li Y, Che M, Bhagat S, Ellis KL, Kucuk O, Doerge DR, Abrams J, Cher ML, Sarkar FH: Regulation of gene expression and inhibition of experimental prostate cancer bone metastasis by dietary genistein. Neoplasia 2004, 6 (4):354-363.

13. Li Y, Sarkar FH: Down-regulation of invasion and angiogenesis-related genes identified by cDNA microarray analysis of PC3 prostate cancer cells treated with genistein. Cancer Lett 2002, 186(2):157-164.

14. Myoung H, Hong SP, Yun PY, Lee JH, Kim MJ: Anti-cancer effect of genistein in oral squamous cell carcinoma with respect to angiogenesis and in vitro invasion. Cancer Sci 2003, 94(2):215-220.

15. Wei H, Saladi R, Lu Y, Wang Y, Palep SR, Moore J, Phelps R, Shyong E, Lebwohl MG: Isoflavone genistein: photoprotection and clinical implications in dermatology. J Nutr 2003, 133(11 Suppl 1):3811S-3819S.

16. Thigpen JE, Locklear J, Haseman JK, Saunders H, Grant MF, Forsythe DB: Effects of the dietary phytoestrogens daidzein and genistein on the incidence of vulvar carcinomas in 129/J mice. Cancer Detect Prev 2001, 25 (6):527-532.

17. Fang MZ, Chen D, Sun Y, Jin Z, Christman JK, Yang CS: Reversal of hypermethylation and reactivation of p16INK4a, RARbeta, and MGMT genes by genistein and other isoflavones from soy. Clin Cancer Res 2005, 11(19 Pt 1):7033-7041.

18. Kikuno N, Shiina H, Urakami S, Kawamoto K, Hirata H, Tanaka Y, Majid S, Igawa M, Dahiya R: Genistein mediated histone acetylation and demethylation activates tumor suppressor genes in prostate cancer cells. Int J Cancer 2008, 123(3):552-560.

19. Majid S, Dar AA, Ahmad AE, Hirata H, Kawakami K, Shahryari V, Saini S, Tanaka Y, Dahiya AV, Khatri G, Dahiya R: BTG3 tumor suppressor gene promoter demethylation, histone modification and cell cycle arrest by genistein in renal cancer. Carcinogenesis 2009, 30(4):662-670.

20. Majid S, Dar AA, Shahryari V, Hirata H, Ahmad A, Saini S, Tanaka Y, Dahiya AV, Dahiya R: Genistein reverses hypermethylation and induces active histone modifications in tumor suppressor gene B-Cell translocation gene 3 in prostate cancer. Cancer 2010, 116(1):66-76.

21. Appleton K, Mackay HJ, Judson I, Plumb JA, McCormick C, Strathdee G, Lee C, Barrett S, Reade S, Jadayel D, Tang A, Bellenger K, Mackay L, Setanoians A, Schatzlein A, Twelves C, Kaye SB, Brown R: Phase I and pharmacodynamic trial of the DNA methyltransferase inhibitor decitabine and carboplatin in solid tumors. J Clin Oncol 2007, 25(29):4603-4609.

22. Barnes S, Peterson TG, Coward L: Rationale for the use of genisteincontaining soy matrices in chemoprevention trials for breast and prostate cancer. J Cell Biochem Supp/ 1995, 22:181-187.

23. Gardner CD, Oelrich B, Liu JP, Feldman D, Franke AA, Brooks JD: Prostatic soy isoflavone concentrations exceed serum levels after dietary supplementation. Prostate 2009, 69(7):719-726.

24. Yegnasubramanian S, Kowalski J, Gonzalgo ML, Zahurak M, Piantadosi S, Walsh PC, Bova GS, De Marzo AM, Isaacs WB, Nelson WG: Hypermethylation of CpG islands in primary and metastatic human prostate cancer. Cancer Res 2004, 64(6):1975-1986.

25. Lodygin D, Epanchintsev A, Menssen A, Diebold J, Hermeking H: Functional epigenomics identifies genes frequently silenced in prostate cancer. Cancer Res 2005, 65(10):4218-4227.

26. Guo L, Zhong D, Lau S, Liu X, Dong XY, Sun X, Yang WW, Vertino PM, Moreno CS, Varma V, Dong JT, Zhou W: Sox7 Is an independent checkpoint for beta-catenin function in prostate and colon epithelial cells. Mol Cancer Res 2008, 6(9):1421-1430

27. Yee DS, Tang Y, Li X, Liu Z, Guo Y, Ghaffar S, McQueen P, Atreya D, Xie J, Simoneau AR, Hoang BH, Zi X: The Wnt inhibitory factor 1 restoration in prostate cancer cells was associated with reduced tumor growth, decreased capacity of cell migration and invasion and a reversal of epithelial to mesenchymal transition. Mol Cancer 2010, 9:162.

28. Bruzzese F, Leone A, Rocco M, Carbone C, Piro G, Caraglia M, Di Gennaro E, Budillon A: HDAC inhibitor vorinostat enhances the antitumor effect of gefitinib in squamous cell carcinoma of head and neck by modulating ErbB receptor expression and reverting EMT. J Cell Physiol 2011, 226 (9):2378-2390

29. Zhau HE, Odero-Marah V, Lue HW, Nomura T, Wang R, Chu G, Liu ZR, Zhou BP, Huang WC, Chung LW: Epithelial to mesenchymal transition (EMT) in human prostate cancer: lessons learned from ARCaP model. Clin Exp Metastasis 2008, 25(6):601-610.
30. McCabe CD, Spyropoulos DD, Martin D, Moreno CS: Genome-wide analysis of the homeobox C6 transcriptional network in prostate cancer. Cancer Res 2008, 68(6):1988-1996.

31. Li LC, Dahiya R: MethPrimer: designing primers for methylation PCRs. Bioinformatics 2002, 18(11):1427-1431.

32. Rothberg JM, Hinz W, Rearick TM, Schultz J, Mileski W, Davey M, Leamon JH, Johnson K, Milgrew MJ, Edwards M, Hoon J, Simons JF, Marran D, Myers JW, Davidson JF, Branting A, Nobile JR, Puc BP, Light D, Clark TA, Huber M, Branciforte JT, Stoner IB, Cawley SE, Lyons M, Fu Y, Homer N, Sedova M, Miao X, Reed B, Sabina J, Feierstein E, Schorn M, Alanjary M, Dimalanta E, Dressman D, Kasinskas R, Sokolsky T, Fidanza JA, Namsaraev E, McKernan K, Williams A, Roth GT, Bustillo $\mathrm{J}$ : An integrated semiconductor device enabling non-optical genome sequencing. Nature 2011, 475(7356):348-352

33. Tusher VG, Tibshirani R, Chu G: Significance analysis of microarrays applied to the ionizing radiation response. Proc Natl Acad Sci USA 2001, 98(9):5116-5121.

34. Jeronimo C, Henrique $R$, Hoque MO, Mambo E, Ribeiro FR, Varzim G, Oliveira J, Teixeira MR, Lopes C, Sidransky D: A quantitative promoter methylation profile of prostate cancer. Clin Cancer Res 2004, 10(24):8472-8478.

35. Singal R, Ferdinand L, Reis IM, Schlesselman JJ: Methylation of multiple genes in prostate cancer and the relationship with clinicopathological features of disease. Oncol Rep 2004, 12(3):631-637.

36. Majid S, Kikuno N, Nelles J, Noonan E, Tanaka Y, Kawamoto K, Hirata H, Li LC, Zhao H, Okino ST, Place RF, Pookot D, Dahiya R: Genistein induces the p21WAF1/CIP1 and p16INK4a tumor suppressor genes in prostate cancer cells by epigenetic mechanisms involving active chromatin modification. Cancer Res 2008, 68(8):2736-2744.

37. Pavese JM, Farmer RL, Bergan RC: Inhibition of cancer cell invasion and metastasis by genistein. Cancer Metastasis Rev 2010, 29(3):465-482.

38. Santibanez JF, Navarro A, Martinez J: Genistein inhibits proliferation and in vitro invasive potential of human prostatic cancer cell lines. Anticancer Res 1997, 17(2A):1199-1204.

39. Dennis G Jr, Sherman BT, Hosack DA, Yang J, Gao W, Lane HC, Lempicki RA: DAVID: Database for Annotation, Visualization, and Integrated Discovery. Genome Biol 2003, 4(5):3.

40. Ingenuity: [http://www.ingenuity.com/]. In

41. Li Y, Kucuk O, Hussain M, Abrams J, Cher ML, Sarkar FH: Antitumor and antimetastatic activities of docetaxel are enhanced by genistein through regulation of osteoprotegerin/receptor activator of nuclear factor-kappaB (RANK)/RANK ligand/MMP-9 signaling in prostate cancer. Cancer Res 2006, 66 (9):4816-4825.

42. Kumi-Diaka J, Merchant K, Haces A, Hormann V, Johnson M: Genistein-selenium combination induces growth arrest in prostate cancer cells. J Med Food 2010 13(4):842-850.

43. Bolos V, Peinado H, Perez-Moreno MA, Fraga MF, Esteller M, Cano A: The transcription factor Slug represses E-cadherin expression and induces epithelial to mesenchymal transitions: a comparison with Snail and E47 repressors. J Cell Sci 2003, 116(Pt 3):499-511.

44. Elloul S, Elstrand MB, Nesland JM, Trope CG, Kvalheim G, Goldberg I, Reich R, Davidson B: Snail, Slug, and Smad-interacting protein 1 as novel parameters of disease aggressiveness in metastatic ovarian and breast carcinoma. Cancer 2005, 103(8):1631-1643.

45. Ohtsuka T, Ishibashi M, Gradwohl G, Nakanishi S, Guillemot F, Kageyama R: Hes 1 and Hes5 as notch effectors in mammalian neuronal differentiation. EMBO J 1999, 18(8):2196-2207.

46. Li X, Martinez-Ferrer M, Botta V, Uwamariya C, Banerjee J, Bhowmick NA: Epithelial Hic-5/ARA55 expression contributes to prostate tumorigenesis and castrate responsiveness. Oncogene 2011, 30(2):167-177.

doi:10.1186/1471-2407-12-145

Cite this article as: Phillip et al:: Genistein cooperates with the histone deacetylase inhibitor vorinostat to induce cell death in prostate cancer cells. BMC Cancer 2012 12:145. 